\title{
Noticias y Comentarios de la UNPHU
}

Por la Lic Irma G. deK undhart Directora del Departamento de Relaciones Públicas

\section{UNPHU.CLAUSURA CURSO INFORMATICA AGRARIA}

El Departamento de Educación Continuada de la Universidad Nacional Pedro Henríquez Ureña clausuró el pasado fin de semana un curso sobre Informática Agraria, en el cual se puso en conocimiento de los profesionales del agro el uso y aplicación del computador en la agricultura, comercialización y uso de los paquetes de programas y microcomputadoras. en el área agrícola.

El curso fue impartido por el Dr.J osé Luis De Miguel Arenal, catedrático de la Escuela Técnica Superior de Ingenieros Agrónomos de la Universidad Politécnica de Madrid y Asesor de Informática del Ministerio de Agronomía de España, quien viajó desde ese país para cumplir compromisos con el Centro Regional de la Oficina Intergubernamental para la Enseñanza de la Informática CREI y la UNPHU.

En la ceremonia de clausura los participantes recibieron sus certificados de asistencia de manos del Rector, Dr.J aime Viñas Román, quien exhortó a los estudiantes a seguir investigando sobre el tema de informática Agraria e indicó que la Universidad seguirá ofreciendo cursos relacionados con la materia en vista de la gran importancia que revela el 'uso de la computadora en la agricultura, para lo cual se mantendrá el contacto con el CREI.

Entre los participantes se encontraban estudiantes de otros países.

Estuvieron presentes en la clausura del curso además del Señor Rector, Dr. Viñas Román, el Vicerrector Académico, Lic. Francisco Polanco Sánchez, el Director del Departamento de Ciencias de la Computadora e Informática, Ing. Pedro Báez y el Director del Plan de Informática, Ing. J osé Guillén. 


\section{UNPHU RENUEVA ACUERDO CON HOSPITAL DE DANBURY}

La Universidad Nacional Pedro Henriquez Ureña y el Hospital de Danbury en Connecticut han renovado un acuerdo de afiliación que fue firmado en el año 1978 entre ambas instituciones. Dicho acuerdo provee posiciones de su-internados en el citado Hospital de Danbury para estudiantes de último año de la Escuela de Medicina de la UNPHU.

El programa se inició en 1974, en base a un acuerdo por medio del cual el Danbury Hospital proveía entrenamiento para tecnólogos médicos de República Dominicana. También contemplaba, y asi se ha hecho, intercambio de profesores de ambas instituciones y talleres-seminarios anuales en el campo de la Tecnología Médica. El Dr. RamónKrawinkel, Subdirector del Departamento de Medicina de Laboratorios es el intermediario responsable para este acuerdo, en esa disciplina.

Luego, en el 1978 se firmó un acuerdo entre el Danbury Hospital y la Escuela de Medicina de la UNPHU, en donde se proveían posiciones de sub-internados para Medicina Interna. De acuerdo con el nuevo programa, Estos sub-internados serán, además de Medicina Interna, en Obstetricia y Ginecología.

Firmaron el actual documento, el Presidente del Hospital Danbury, Dr.J ohn Creasy y el Dr. Mariano Defillé, Ricart, Director de la Escuela de Medicina de la UNPHU.

\section{UNPHU CONTINUA DISTRIBUCION DE ARBOLES FRUTALES Y MADERABLES EN SECCIONES ALEDAÑAS A LA HACIENDA NIGUA.}

Dentro de los lineamientos de la campaña que lleva a cabo la Universidad Nacional Pedro Henríquez Ureña para repoblar con árboles frutales y maderables las secciones y parajes aledañas a su Recinto Agropecuario de la Hacienda Nigua, personal técnico de esa institución procedió a la distribución y siembra en el paraje de "Montaño." de 1,131 árboles frutales, distribuídos de la siguiente manera

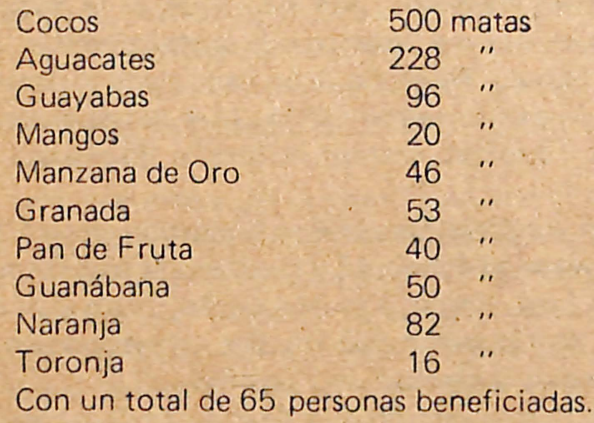


En la sección de Malpaez se distribuyeron 1,500 árboles frutales, distribuidos así:

$\begin{array}{lr}\text { Cocos } & 678 \text { matas } \\ \text { Aguacate } & 285 \\ \text { Guayabas } & 132 \text { " } \\ \text { Mango } & 57 \text { " } \\ \text { Manzana de Oro } & 65 \text { "' } \\ \text { Granada } & 37 \text { " } \\ \text { Pan de Fruta } & 67 " \\ \text { Guanábana } & 53 \text { " } \\ \text { Naranja } & 53 \text { " } \\ \text { Toronja } & 45 \text { " } \\ \text { Cajuil } & 5\end{array}$

Se han beneficiados 71 moradores de la mencionada sección.

NUEVA DISTRIBUCION Y SIEMBRA POR PARTE DE LA UNPHU DE FRUTALES EN LOS ALREDEDORES DE LA HACIENDA NIGUA.

Continuando las labores de siembra de árboles maderables y frutales, personal técnico de la UNPHU recientemente distribuyó y fueron sembrados:

A la Asociación "La Luchadora", de Sabana Grande de Palenque: 1 , 671 plantas, distribuídas de la siguiente manera: 804 cocos; 260 aguacates de variedades tardías, 126 pan de frutas, 52 de guanábanas, 117 de guayabas, 42 de jagua, 67 de tamarindo, 126 de mangos banilejos, 31 de naranjas valencianas, 36 de manzana de oro, y 10 de cajuil. A cada uno de los 42 socios de la citada asociación, le correspondieron 41 plantas.

También en el Municipio de Sabana Grande de Palenque, a la Cooperativa "Amantes del Progreso", con 50 socios, se le distribuyeron 1, 200 plantas, de las cuales a cada socio le correspondieron 24.

En la Sección Sabana Palenque, a la Asociación Agropecuaria "La Esperanza", se le distribuyeron 794 frutales de las especies ya señaladas, entre los 33 socios, correspondiéndole 25 plantas a cada uno de ellos.

A la Asociación Agrícola Palenque se le distribuyeron 1,150 plantas de frutales, correspondiéndole 45 a cada uno de los 30 socios de ella. En la Sección J uan Barón, a la Asociación "J uan Barón Unido en Marcha", se le distribuyeron 568 frutales, correspondiéndole a cada uno de los 24 socios la candiad de 25 plantas.

También en esa misma sección a la Asociación "J uan Barón Unido" 
se le distribuyeron 1,056 plantas, y a sus 44 socios le correspondieron 24 plantas a cada uno.

En total fueron sembrados 6,394 árboles frutales entre las asociaciones correspondientes a Sabana Grande de Palenque, Sabana Palenque y J uan Barón.

\section{TECNICOS DE LA UNPHU REGRESAN DE VIAJ E DE ENTRENA- MIENTO EN FRANCIA}

A mediado de septiembre arribaron al país los Dres. Pelayo Mallén Cuesta y Sandra Moré Guaschino, Director y Subdirectora, respectivamente, del Laboratorio de Procesamiento de Semen de la UNPHU, en su Recinto Agropecuario de Nigua.

Los citados profesionales pasaron cerca de dos meses en un curso dictado conjuntamente entre la firma IMV (Instruments Médicine Vetérinaire) y el INRA (Instituto Nacional de Investigación Agropecuaria), el cual fue dictado en L'Aigle, una población cercana a París donde están ubicados los laboratorios de la firma IMV.

En ese curso de carácter teórico práctico, dichos profesionales aprendieron a cabalidad la técnica de cómo realizar la transferencia de embriones en bovinos, con el propósito de que a su regreso, en el país se ponga en ejcución este sistema novedoso de reproducción animal.

El curso que tenía carácter internacional, y al cual asistieron profesionales de Argentina, Mejico, España y Cuba, el Dr. Pelayo Mallén Cuesta obtuvo las notas más altas en el mismo, según información que le suministra el IMV a la UNPHU.

Una vez realizado dicho curso, los profesionales efectuaron una gira por distintas localidades de Francia para visitar centros de inseminación artificial, cooperativas agropecuarias $y$ estaciones de investigación ganadera, atendiendo a una invitación que les formulara el señor Daniel Parrot, de la Oficina para el Desarrollo de la Producción Agrícola del Gobierno de Francia, quien había visitado con anterioridad nuestro país.

El equipo para realizar las técnicas de transferencia de embriones fue adquirido por la Universidad Nacional Pedro Henríquez Ureña, y ya llegó al país. Tan pronto se termine la construcción del edificio de ese laboratorio, se procederá a la instalación del mismo, lo cual se espera que será a fines del presente año.

En los primeros meses del próximo año, el INRA enviará uno de sus técnicos más capacitados para realizar demostraciones de transplante de embriones en el Recinto Agropecuario de la Hacienda Nigua, de manera que nuestros profesionales Médicos Veterinarios y de producción Animal, asi como los ganaderos en general, puedan apreciar la importancia y el alcance de esta novedosa técnica de reproducción animal que está revolucionando la ganaderia moderna. 
El Gobierno de Israel, a través de la Embajada de ese país en el nuestro, ha otorgado una beca a la DRA. ANA MARIA PEPEN LUNA, Médico Veterinaria del Centro de Reproducción Ganadera de la UNPHu, para participar en el Curso Internacional sobre Cría, Nutrición y Administración de Ganado Lechero, que tendrá lugar del 10 de octubre al 10 de diciembre del presente año en elK ibutz Shefayim.

La Dra. Pepén Luna trabaja con el ganado lechero de la Hacienda de Nigua y durante el curso que va a recibir residirá en el citadoK ibutz para que de manera práctica y objetiva adquiera los conocimientos relativos al manejo del ganado lechero.

La UNPHU le ha dado especial importancia a este entrenamiento, pues sabe que el ganado Holstein de Israel figura en primer lugar a nivel mundial, en lo que se refiere a productividad, estando el promedio de producción de las vacas Holstein israelies por encima del que se obtiene en Holanda, Inglaterrà, Canadá y Estados Unidos, y estimando que entrenamientos de esta naturaleza contribuirán notablemente a mejorar el grado de preparación de los técnicos que trabajan en el Centro de Reproducción Ganadera en la Hacienda Nigua.

\section{UNPHU RECIBE DONACION MUESTRA PICTORICA CARIMOS}

Recientemente el Vicerrector Académico de la Universidad Nacional Pedro Henriquez Ureña, en funciones de Rector, recibió en donación copias de la muestra pictórica de la Arquitectura Vernácula del Gran Caribe que fue presentada con ocasión de la Primera Conferencia Interamericana Preparatoria sobre la Conmemoración del V Centenario del Descubrimiento de América, evento que se realizó en nuestra ciudad capital.

La mencionada exposición pictórica fue organizada en aquella ocasión por la OEA y la UNPHU a través del Ilamado "Plan del Gran caribe para los Monumentos y Sitios" (CARIMOS), y presentaba magníficas representaciones fotográficas de la arquitectura tradicional de la zona del Caribe. La muestra estuvo entonces localizada en la Catedral de Santo Domingo, y fue inaugurada oficialmente por Su Santidad J uan Pablo II en su segunda visita a la Basílica Metropolitana Primada de América, el dia 11 de octubre de 1984 .

Los arquitectos Eugenio Pérez Montás y Esteban Prieto estuvieron presentes en el acto de entrega de la muestra al Vicerrector Polanco, $y$ fueron ellos quienes la presentaron a la UNPHU oficialmente en nombre de CARIMOS. 


\section{UNPHU FIRMA ACUERDO CON FONDOPREI}

La Universidad Nacional Pedro Henríquez Ureña y el Fondo Dominicano de Preinversión (FONDOPREI) firmaron en el día de ayer, jueves 14 de junio, un nuevo contrato de préstamo, concertado para la realización de los estudios "Factibilidad para la instalación de un Centro Nacional de Pesca" y "Factibilidad para la instalación de una planta para la elaboración de conservas de frutas y vegetales". EI FONDOPREI formaliza la tercera y cuarta transación crediticia en los últimos dos años, en favor de la UNPHU.

Los anteriores créditos del Fondo a la UNPHU fueron otorgados para la elaboración del "Estudio de Factibilidad para la instalación de un Laboratorio de Semen Congelado" así como de los "Estudios para el proyecto de desarrollo y adiestramiento pecuario de la Hacienda Nigua."

Precisamente, el Proyecto de Isstalación del Laboratorio de Semen Congelado se encuentra actualmente en pleno proceso de operación, proporcionando servicios principalmente a pequeños y medianos ganaderos, a través de acciones directas, así como indirectamente por medio de los programas oficiales de inseminación artificial, ya que la UNPHU suministra este material reproductivo a la Secretaría de Estado de Agricultura (SEA).

Los estudios concernientes al Proyecto de Desarrollo y Adiestramiento Pecuario de la Hacienda Nigua, en estos momentos se encuentra en sus fases finales de elaboración.

\section{INVESTIGACION DE FINCAS}

Visita de nuevo la Universidad Nacional Pedro Henriquez Ureña el Dr. R. E. McDowell, Director de la Escuela de Producción Animal de la Universidad de Cornell con el propósito de asesorar un proyecto de investigación en fincas integradas.

Las investigaciones se harán en todas las áreas de ganado de doble propósito, producción de carne y leche, en el cual se incluyen actividades sobre nutrición, reproducción, manejo animal, pastos y forrajes, aspectos económicos, etc. La implementación del mismo se hará en base a una encuensta pecuaria a nivel nacional preparada por la UNPHU con el propósito de diagnosticar los principales problemas que confrontan los productores actualmente.

EI D. McDowell es profesor internacional de Producción Animal, Asesor del Departamento de Agricultura de los Estados Unidos y autor de varias obras sobre ganadería tropical que han alcanzado difusión mundial, por lo que se le considera una de las más altas autoridades a nivel interna- 
cional. Sus visitas han sido fecundas y provechosas dada la vasta experiencia y el conocimiento en los asuntos agropecuarios en los cuales está empeñada la UNPHU, camino a su plan de establecer una Universidad Agraria como cosa prioritaria en nuestro país.

UNPHU GANA MAXIMOS HONORES EN LA FERIA RADIO ALEGRE DE SANTIAGO' R.D.

Los ejemplares del Centro de Reproducción Ganadera de la UNPHU del Recinto Agropecuario de Nigua, ganaron los premios máximos en las diferentes competencias que tuvieron lugar durante el transcurso de la Feria Radio Alegre, recién celebrada en Santiago.

Asi, la UNPHU obtuvo en la Raza Holstein los premios de Campeón Adultos y Gran Campeón.

En la raza Pardo Suiza, Reservado de Campeón Adulto y Gran Campeón. Y con su ejemplar 209 obtuvo el galardón del Mejor Ejemplar Puro de las Razas Lecheras.

En la raza Brahman conquistó el galardón de Reservada de Campeona J oven y Reservado de Campeón Adulto, y la Mejor Descendencia de un Reproductor, correspondiéndole ese honor a su famoso toro Moscú.

En la raza Indu Brasil conquistó el Reservado de CampeónJ oven, el de Campeón Adulto y el de Gran Campeón. Estos dos últimos premios con su ejemplar Coyote. También en toro Coyote obtuvo el premio del Mejor Ejemplar de las Razas de Carne.

Un ejemplar hembra de la raza Santa Gertrudis, ganó el Mejor Ejemplar de Carne.

Por todos los premios conquistados en las distintas razas, la UNPHU fue declarada el Mejor Expositor de la Feria.

\section{CENA CHARLA DE EGRESADOS UNPHU}

Los egresados de la Facultad de Ciencias Agropecuarias y Recuros Naturales de la UNPHU celebraron una Cena-Charla, el sábado 27 de agosto en el Hotel Comodoro, la cual tuvo como orador invitado al Dr.J aime A. Viñas Román, Rector de la Universidad Nacional Pedro Henriquez Ureña

E) Dr. Viñas trató acerca de: "La Educación Rural, la Reforma Agraria, el Desarrollo Agropecuario y la Universidad Nacional Pedro Henriquez Ureña en la República Dominicana."

En su tema, el orador invitado desarrolló importantes aspectos de la reforma agraria nuevos modelos o nuevas alternativas. 


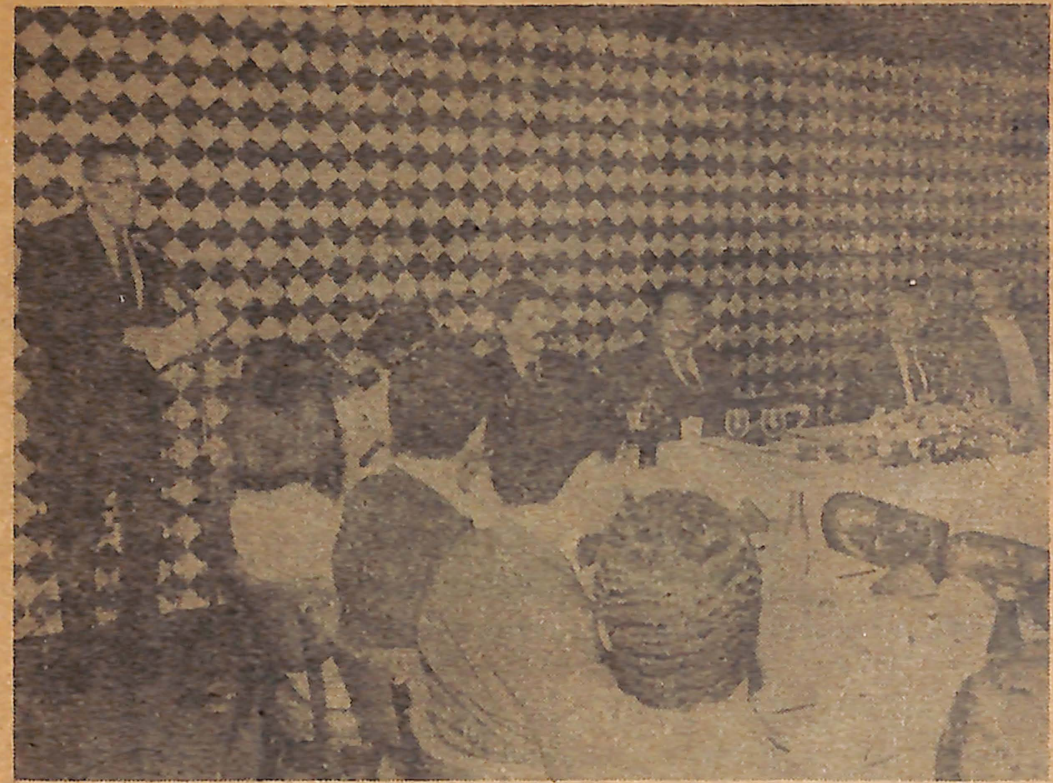

Cona do Egreindos da Agropacuarla.

Uno de los motivos que ha movido a la Sociedad de Egresados a ofrecer esta cena-charla es manifestar su preocupación por la situación que hace algún tiempo amenaza la tranquilidad del Recinto Agropecuario UNPHU en torno a la integridad de las tierras en donde ha de tener asiento la Universidad Agraria que tanto beneficio traerá al país.

\section{UNPHU CONTRATA EXPERTA BRASILEÑA EN CURRICULUM}

La Universidad Nacional Pedro Henríquez Ureña en colaboración con el Comité Intergubernamental para Migraciones (CIM) ha contratado a la Dra. Lady Lina Traldy, experta en Curriculum, quien realizará trabajos de investigación, desarrollará cursos especiales y asesorará al Departamento de pedagogía, así como a la Vicerrectoría Académica y a la Universidad de Superación y Evaluación Profesoral.

En la actualidad, a través del CIM, institución de carácter internacional dedicada al intercambio de expertos, con sede en Ginebra, la UNPHU ha logrado también los servicios de un especialista en Foresta, el Ing. Patricio Santa Cruz, quien está dando asesoría a los proaramas de Recursos Natural en que está empeñado, tanto la Universidad como el Gobierno. 


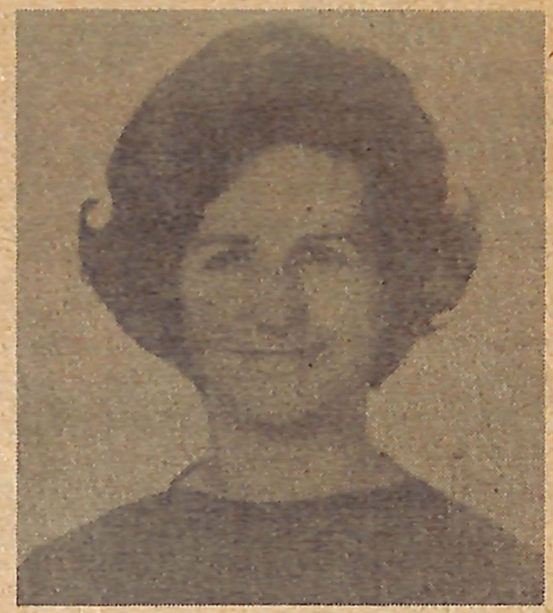

Dra. Lady Lima Traldy

La Dra. Traldy es una distinguida educadora brasileña, doctorada en Pedagogía en la Universidad de Sao Paulojy con Maestría en Educación en George Peabody College for Teachers y Universidad de Indiana, en los Estados Unidos. Es fundadora y ex-Directora de la Facultad de Educación de la Universidad de Brasilia.

Entre otros cargos y funciones importantes que ha desempeñado la Dra. Traldy se pueden señalar los de Titular del Curso Interamericano de Especialistas de Ciencias-Desarrollo de Curriculum de la Universidad de Sao Paulo; Directora del Centro de Post-Grado del Núcleo Universitario de Santo André, S. P.; Titular de curriculos y programas, curso de Maestría, Universidad Federal de Río deJ aneiro y Experta de la OEA en Curriculos en el Curso Internacional, desarrollado en Caracas, Venezuela.

En relación con trabajos publicados por la profesora Lady Lina Traldy, merecen atención: Curriculo, Desarrollo de Curriculo, Un Ideal en Educación, La Orientación Educacional y sus Relaciones con la Dirección, etc.

\section{ASOCIACIONES GANADERAS DE LA ZONA FRONTERIZA NORTE VISITAN LA HACIENDA NIGUA}

Un grupo de unos 60 ganaderos de las asociaciones correspondientes a Dajabón, Loma de Cabrera, Partido y Copey, asistieron a un "Día de Campo" ofrecido por la UNPHU en su Recinto Agropecuario de la Hacienda Nigua.

La citada visita constituyó una revelación para esos criadores, porque en el transcurso de la misma pudieron observar las modenras instalaciones 
que esa universidad tiene alli, y en ese sentido visitaron el establo lechero con sus distintas dependencias, el laboratorio de procesamiento de semen congelado, los toriles donde están alojados los mejores reproductores de las razas Holstein, Pardo Suiza, Charolais, Santa Gertrudis, Brahman y Simmental, y los hatos de esas distintas razas que suman más de 1,000 cabezas, así como el vivero de árboles frutales y maderables que la UNPHU tiene en su Campo de práctica Experimental para la repoblación de las secciones y zonas aledañas a la Hacienda Nigua, en las cuales viene desarrollando de una manera sistemática desde hace más de un año, la siembra de aguacates injertos de variedades tardías, distintas variedades de cítricos, tamarindo, mangos seleccionados, zapote, guayabas, pan de fruta, manzana de oro, etc., así como cocos híbridos obtenidos en Samaná y árboles maderables como Caoba, Corazón de Paloma, Acacia Amarilla, Eucaliptus y Pinos.

\section{UNPHU CELEBRARA CENTENARIO NACIMIENTO MAX HENRIQUEZ UREÑA.}

La Universidad Nacional Pedro Henríquez Ureña "UNPHU" a través de su Consejo Académico, decidió celébrar el centenario del nacimiento del gran humanista dominicano Max Henríquez Ureña, quien fuera su profesor hasta el momento de su muerte, que le sorprendió como a su hermano Pedro camino a su deber docente. Max Henríquez Ureña nació el 16 de noviembre de 1985 y dejó una obra fecunda que honra la bibliografía hispanoamericana y de hondo contenido dominicanista.

La UNPHU ha formado una comisión que preside el Dr. Próspero Mella Chavier, Vicerrector Administrativo, la cual tiene a su cargo coordinar los diferentes actos de tan magna conmemoración.

Los otros miembros de la comisión son : Dr. Mariano Lebrón Saviñón, Director de Publicaciones; Lic. Irma Grullón deK unhardt, Directora de Relaciones Públicas; Lic. Edgar Senior, Secretario del Consejo Académico y cuenta con la asesoría del Dr.J osé Henríquez Almánzar.

\section{UNPHU HONRA PEDRO HENRIQUEZ UREÑA.}

"Estamos cumpliendo con un compromiso contraído por la Universidad Nacional Pedro Henriquez Ureña, al celebrar actos como éste todos los 29 de junio", fueron las palabras iniciales del Dr.J aime Viñas Román. Rector de la UNPHU, en una ofrenda floral, ofrecida por la Institución a Pedro Henríquez Ureña con motivo de su fecha natilicia.

Dicha ofrenda se llevó a efecto a las 11.00 a.m., frente al busto del gran humanista, en el Recinto I de la UNPHU. 


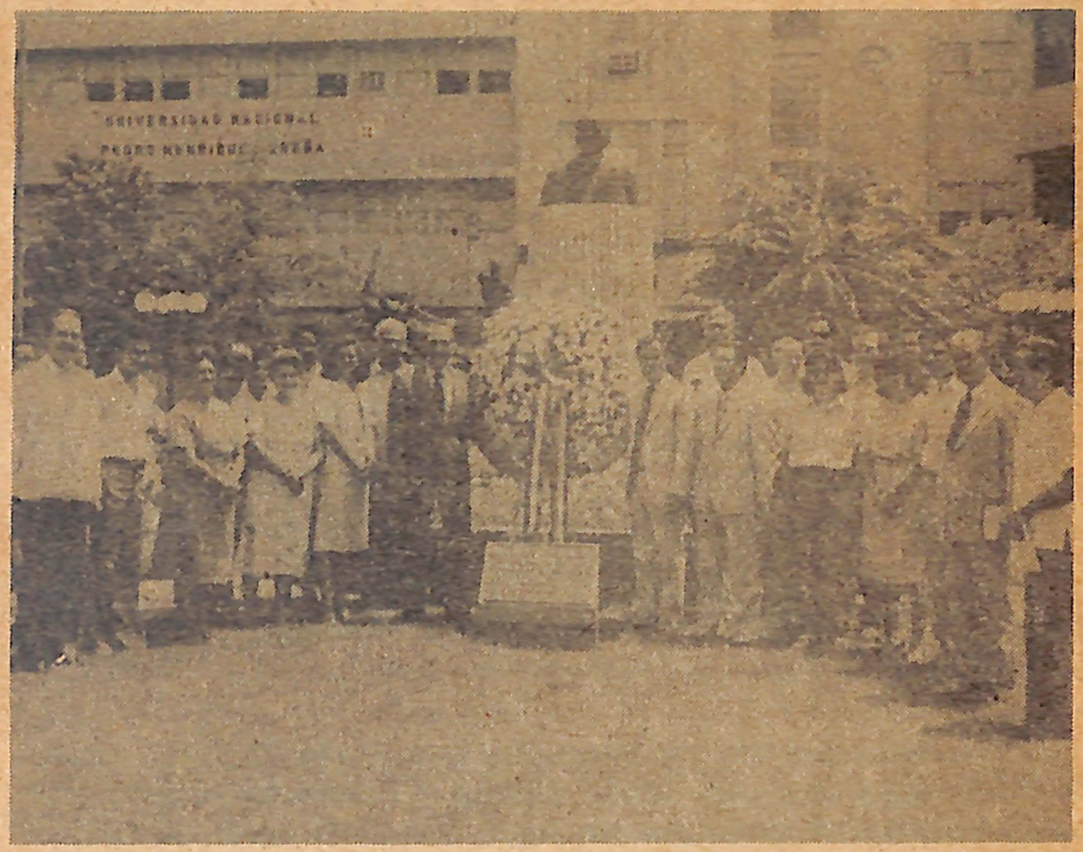

Ofrenda Fioral ante el Busto de Pedro Henríquez Ureña en Recinto i de ta UNPHU.

El Dr. Viñas destacó, en las breves palabras pronunciadas en el momento de la ofrenda, la labor educacional de este gran dominicano, que ha sido comparada con la de otros grandes de América como Bello y Sarmiento.

Enfatizó que la UNPHU tiene dos fechas históricas en las cuales se hace riguroso una ofrenda floral, primero en enero, el aniversario del natalicio del Padre de la Patria, J uan Pablo Duarte, cuando la comunidad se hace presente ante la estatua heróica del Pațicio y la segunda, en junio ante el Busto de Pedro Henríquez Ureña.

También el Rector recordó la vehemencia con que la UNPHU celebró el Año Centenario del nacimiento del hombre que es considerado el Primer Humanista del Continente, con actos que culminaron hace precisamente un año, dejando un recuerdo en el ámbito de nuestra Universidad. .

Además del Dr. Viñas Román, se encontraban presentes en el homenaje a Pedro Henríquez Ureña, los vicerrectres Administrativo y de Desarrollo, Dr. Próspero Mella Chavier y Arq. Roberto Bergés, respectivamente; decano $y$ directores de escuelas, departamentos y programas, asi como profesores y estudiantes. 
Emilio Carilla, argentino, Profesor Emérito de la Universidad de Tucumán y reconocido humanista hispanoamericano - quién fuera discipulo de Pedro Henríquez Ureña, el humanista dominicano de estatura continental -, fue el ganador del Premio en el Concurso Internacional que sobre "Pensamiento y la Obra de Pedro Henríquez Ureña" convocó la Organización de los Estados Americanos, (OEA) y la Universidad Nacional Pedro Henríquez Ureña (UNPHU), con motivo del centenario de la muerte del relevante americano. El premio obtenido por el Sr. Carilla consiste en un diploma, la publicación de la obra ganadora y cinco mil dólares.

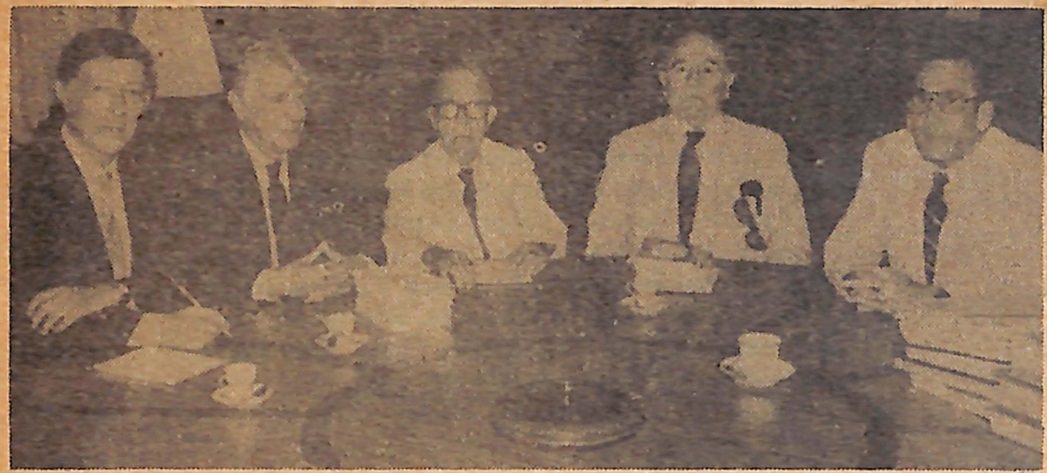

Los Sres. Integrantes del jurado, mientras deliberabam para dotorminar ol canedor dol concurso sobre la vida y abra de Pedro Henríquez Urefla, organizado por la OEA con motivo de cumplirse, el año pasado, el centenario del natalicio de este humanista. So observan en la foto: ei Dr. Jaime Viñas Román, Rector de la UNPHU; Sr. Leopoldo Zea, de México; Sr. Delfín Leocadio Garasa, de Argentina; Sr. Pedro Troncoso Sánchez y el Lic. Emilio Rodriguez Demorizi, de República Dominicana.

Un jurado Internacional reunido en la UNPHU, presidido por el Rector de esta institución docente, Dr.J aime Viñas Román y compuesto por el Lic. Emilio Rodríguez Demorizi, Presidente de la Academia Dominicana de la Historia y el Lic. Pedro Troncoso Sánchez, Presidente de la Academia Dominicana de Ciencias, y los doctores Leopoldo Lea, Profesor de la Universidad Autónoma de México y Delfin Leocadio Garaza Profesor de la Universidad de Buenos Aires, decidió por unanimidad proponer para el Primer Premio el estudio "Pedro Henríquez Ureña. Signo de América", por su abarcadora información bibliografica, su sentido crítico y por destacar el espíritu americanista que animó la obra del maestro. Se decidió, asimismo, otorgar menciones honoríficas a los trabajo presentados por los conocidos escritores latinoamericanos, Enrique Zuleia Alvarez, de Argentina, Alberto Baeza Flores, de Chile y a la profesora Laura Febres de Ayala, de Venezuela 
Esta publicaciốn se imprimió en los Talleres Offset de la Universidad Nacional Pedro Henriquez Ureña: Encargado: Genaro Phillips; Composición tipográfica: Vicente Salas Woss Diagramador. Máximo Garcfa: Fotomecánica: Francisco Tavårez; Impresiőn: Nelson Veloz, y Carlos M. Rodrfguez: Compaginación y Encuadernación: Eury Hernåndez, Juan Prenza y Ramón A. Marte. 